\title{
ON THE ASYMPTOTIC BEHAVIOR OF KAC-WAKIMOTO CHARACTERS
}

\author{
KATHRIN BRINGMANN AND AMANDA FOLSOM
}

(Communicated by Ken Ono)

\begin{abstract}
Recently, Kac and Wakimoto established specialized character formulas for irreducible highest weight $s \ell(m, 1)^{\wedge}$ modules and established a main exponential term in their asymptotic expansions. By different methods, we improve upon the Kac-Wakimoto asymptotics for these characters, obtaining an asymptotic expansion with an arbitrarily large number of terms beyond the main term. More specifically, it is well known that in the case of holomorphic modular forms, asymptotic information may be obtained using modular transformation properties. However, here this is not the case due to the analytic nature of the Kac-Wakimoto series as discovered recently by the first author and Ono. We first "complete" these series by adding to them certain integrals, obtaining functions that exhibit suitable modular transformation laws, at the expense of the completed objects being nonholomorphic. We then exploit this mock-modular behavior of the Kac-Wakimoto series to obtain our asymptotic expansion. In particular, we show that beyond the main term, the asymptotic behavior is dictated by the nonholomorphic part of the completed Kac-Wakimoto characters, which is a priori invisible. Euler numbers (equivalently, zeta-values) appear as coefficients.
\end{abstract}

\section{INTRODUCTION AND STATEMENT OF RESULTS}

One of the most beautiful connections between modular forms and Lie algebras is given by "Monstrous moonshine". In 1979, Conway and Norton made a then surprising conjecture relating the Fourier coefficients of the modular function $j$, defined by

$$
j(\tau)=\frac{1}{q}+744+196884 q+21493760 q^{2}+\ldots
$$

$\left(q:=e^{2 \pi i \tau}, \tau \in \mathbb{H}\right)$, to dimensions of irreducible representations of the Monster group [8], and Borcherds ultimately gave a proof in 1992 [1]. Prior to this, important work of Kac 12 established the so-called Weyl-Kac character formula and denominator identity, first relating infinite-dimensional Lie algebras and modular forms. Of the numerous consequences of Kac's work are many beautiful identities. For example, if we let

$$
q^{\frac{d}{24}} \sum_{n \geq 0} p_{d}(n) q^{n}:=q^{\frac{d}{24}} \prod_{k \geq 1}\left(1-q^{k}\right)^{d}
$$

Received by the editors September 5, 2011.

2010 Mathematics Subject Classification. Primary 11F22, 17B67, 11 F37. 
denote the $d$-th power $(d \in \mathbb{Z})$ of the Dedekind $\eta$-function, $\eta(\tau):=q^{\frac{1}{24}} \prod_{n \geq 1}\left(1-q^{n}\right)$ (a weight $\frac{1}{2}$ modular form), the Weyl-Kac denominator formula and the "MacDonald identities" established that the coefficients $p_{d}(n)$ encode information about representations of affine Lie algebras [19]. Others, including Lepowsky, Milne and Wilson [16, 17, 18, proved further identities along these lines and showed, for example, that the classical Rogers-Ramanujan identity

$$
\sum_{n \geq 0} \frac{q^{n^{2}}}{(q ; q)_{n}}=\prod_{n \geq 1}\left(1-q^{5 n-1}\right)^{-1}\left(1-q^{5 n-4}\right)^{-1},
$$

$(a ; q)_{n}:=\prod_{j=0}^{n-1}\left(1-a q^{j}\right)$, and subsequent generalizations become principally specialized characters for the standard modules for $A_{1}^{(1)}$.

More recently, Kac and Wakimoto [14 found a specialized character formula for $\operatorname{tr}_{L\left(\Lambda_{(s)}\right)} q^{L_{0}}$, where $L\left(\Lambda_{(s)} ; r+1\right)$ is the irreducible $s \ell(m, 1)^{\wedge}$ module with highest weight $\Lambda_{(s)}$, and $L_{0}$ the "energy operator". In [7], the first author and Ono answered a question of Kac regarding the "modularity" of the characters $\operatorname{tr}_{L\left(\Lambda_{(s)}\right)} q^{L_{0}}$ and proved that they are "holomorphic parts" of certain nonholomorphic modular functions. Following this, in [10, the second author showed that the Kac-Wakimoto characters are, up to simple $q$-series, universal mock theta functions [11.

In this paper, we aim to understand the asymptotic behavior (see [13]) of the Kac-Wakimoto characters of [14], given by

$$
\operatorname{tr}_{L\left(\Lambda_{(s)}\right)} q^{L_{0}}:=2 q^{-\frac{s}{2}} \frac{\phi\left(q^{2}\right)^{2}}{\phi(q)^{m+2}} \sum_{k=\left(k_{1}, \ldots, k_{m-1}\right) \in \mathbb{Z}^{m-1}} \frac{q^{\frac{1}{2} \sum_{i=1}^{m-1} k_{i}\left(k_{i}+1\right)}}{1+q^{\sum_{i=1}^{m-1} k_{i}-s}},
$$

with $s \in \mathbb{Z}, m \geq 2, m \in \mathbb{N}$, and $\phi(q):=\prod_{n=1}^{\infty}\left(1-q^{n}\right)$.

Replacing $\tau$ by it, $t \in \mathbb{R}^{+}$, Kac and Wakimoto [14] established the following asymptotic behavior for $\operatorname{tr}_{L\left(\Lambda_{(s)}\right)} q^{L_{0}}$ as $t \rightarrow 0^{+}$:

$$
\operatorname{tr}_{L\left(\Lambda_{(s)}\right)} q^{L_{0}} \sim \frac{\sqrt{t}}{2} e^{\frac{\pi(m+1)}{12 t}} .
$$

Their methods involve series manipulations and modular transformations of theta functions.

Using completely different methods, our main result in this paper improves upon the asymptotic behavior given for $\operatorname{tr}_{L\left(\Lambda_{(s)}\right)} q^{L_{0}}$ as established by Kac and Wakimoto in (1.2), who give the main exponential term in the asymptotic expansion of $\operatorname{tr}_{L\left(\Lambda_{(s)}\right)} q^{L_{0}}$. Here, we obtain an asymptotic expansion for $\operatorname{tr}_{L\left(\Lambda_{(s)}\right)} q^{L_{0}}$ with an arbitrarily large number of terms (beyond the main term). More precisely, it is well known that in the case of holomorphic modular forms, asymptotic information may be understood using modular transformation properties. However, here, due to the analytic nature of the Kac-Wakimoto series, as discovered recently by the first author and Ono [7, we must proceed by different methods. We first relate the Kac-Wakimoto characters $\operatorname{tr}_{L\left(\Lambda_{(s)}\right)} q^{L_{0}}$ to "multivariable Appell functions", natural generalizations of certain Lerch sums studied recently by Zwegers 21, 22. (see §2 for definitions). Zwegers' work, together with the work of Ono and the first author have sparked a flurry of recent research on harmonic weak Maass forms and mock theta functions (see for example [2, 3, 4, 5, 6, 20]). We point out the historical significance of Zwegers' thesis [21, which after nearly 100 years finally explained the 
"modularity" of Ramanujan's mock theta functions (simple $q$-series expansions resembling classical theta functions) via the mock-modular functions $\mu(u, v ; \tau)$. (See (2.)

We then exploit the philosophy that mock modular forms may be "completed" by adding certain nonholomorphic integrals to produce functions exhibiting suitable modular transformation properties, at the expense of the completed objects being nonholomorphic. We show that in the case of the Kac-Wakimoto series, beyond the main term, their asymptotic behavior is dictated by the nonholomorphic part of the completed series, which is a priori invisible.

To state our theorem, we let $E_{j}, j \geq 0$, denote the Euler numbers (relatives of Bernoulli numbers), defined by the generating function

$$
\sum_{j=0}^{\infty} \frac{E_{j} x^{j}}{j !}=\frac{2}{e^{x}+e^{-x}}
$$

Theorem 1.1. Let $m, N \in \mathbb{N}, m \geq 2$, and $s \in \mathbb{Z}$. Then as $t \rightarrow 0^{+}$, we have

$$
\operatorname{tr}_{L\left(\Lambda_{(s)}\right)} q^{L_{0}}=\frac{\sqrt{t}}{2} e^{\frac{\pi(m+1)}{12 t}-\frac{\pi(m-2) t}{12}}\left(\sum_{j=0}^{N} a_{j}(m-1, s) t^{j}+O\left(t^{N+1}\right)\right),
$$

where the coefficients $a_{j}(n, s), n \in \mathbb{N}, s \in \mathbb{Z}$ are defined as

$$
a_{j}(n, s):=\frac{(\pi s)^{j}}{j !} \sum_{\ell=0}^{j}\left(\begin{array}{l}
j \\
\ell
\end{array}\right)\left(\frac{n}{4 s}\right)^{\ell} E_{j+\ell} .
$$

Further, if $m-1=2 s$, we have as $t \rightarrow 0^{+}$,

$$
\operatorname{tr}_{L\left(\Lambda_{(s)}\right)} q^{L_{0}}=\frac{\sqrt{t}}{2} e^{\frac{\pi(m+1)}{12 t}+\frac{\pi t(5-4 m)}{12}}+O\left(e^{\frac{\pi\left(m^{2}-12 m+14\right)}{12 t}}\right) .
$$

Remarks.

1) The implied constant in Theorem 1.1 depends on $N, m$, and $s$.

2) Conversation and correspondence with Don Zagier led us to believe that the case $n=2 s$ (see (1.4) and (3.3)) is indeed special. More specifically, by ellpitic transformation laws satisfied by the Mordell integral $h(u ; \tau)$ (see $\S 2$ ), it would be sufficient to assume $0 \leq s<n$. Zagier's work to us implied that in this range, the case $n=2 s$ is the only setting in which the $a_{j}(n, s)$ are expected to have exponential growth.

3) We show in the course of the proof of Theorem 1.1 that the terms $\exp (-\pi(m-2) t / 12) a_{j}(m-1, s) t^{j}$ are dictated by the nonholomorphic part of the completed nonholomorphic Kac-Wakimoto series.

\section{Multivariable Appell functions and modularity of KAC-WAKIMOTO CHARACTERS}

To study the asymptotic behavior of the Kac-Wakimoto characters $\operatorname{tr}_{L\left(\Lambda_{(s)}\right)} q^{L_{0}}$ we would like to map the cusp 0 to the cusp $\infty$. For this, we rewrite the series in terms of multivariable Appell functions, which are related to automorphic objects, and were recently studied by Zwegers 21,22 . We start by recalling these functions 
and their automorphic properties. Firstly define for $n \in \mathbb{N}$,

$$
\mu_{n}(u, v)=\mu_{n}(u, v ; \tau):=\frac{e^{\pi i u}}{\prod_{j=1}^{n} \vartheta\left(v_{j} ; \tau\right)} \sum_{k \in \mathbb{Z}^{n}} \frac{(-1)^{|k|} q^{\frac{1}{2}|| k||^{2}+\frac{1}{2}|k|} e^{2 \pi i k \cdot v}}{1-e^{2 \pi i u} q^{|k|}}
$$

where $|k|:=\sum_{i=1}^{n} k_{i},\|k\|^{2}:=\sum_{i=1}^{n} k_{i}^{2}$, and $u \in \mathbb{C}, v \in \mathbb{C}^{n}, \tau \in \mathbb{H}$. The classical Jacobi theta function $\vartheta$ is defined by

$$
\begin{aligned}
\vartheta(u) & =\vartheta(u ; \tau):=\sum_{\nu \in \frac{1}{2}+\mathbb{Z}} e^{\pi i \nu^{2} \tau+2 \pi i \nu\left(u+\frac{1}{2}\right)} \\
& =-i q^{\frac{1}{8}} e^{-\pi i u} \prod_{n=1}^{\infty}\left(1-q^{n}\right)\left(1-e^{2 \pi i u} q^{n-1}\right)\left(1-e^{-2 \pi i u} q^{n}\right) .
\end{aligned}
$$

To state the transformation laws of $\mu_{n}$, we require further functions. To be more precise, we define "shifted" multivariable Appell sums $\mu_{n, \ell}$ and also a Mordell integral $h$. These functions are given by $(0 \leq \ell \leq n-1)$

$$
\begin{gathered}
\mu_{n, \ell}(u, v)=\mu_{n, \ell}(u, v ; \tau):=(-1)^{\ell} q^{-\frac{\ell^{2}}{2 n}} e^{-\frac{2 \pi i \ell}{n}(u-|v|)} \mu_{n}(u+\ell \tau, v ; \tau), \\
h(u)=h(u ; \tau):=\int_{\mathbb{R}} \frac{e^{\pi i \tau x^{2}-2 \pi u x}}{\cosh (\pi x)} d x .
\end{gathered}
$$

Clearly we have that $\mu_{n}(u, v)=\mu_{n, 0}(u, v)$. The following proposition gives an expression for the Kac-Wakimoto characters $\operatorname{tr}_{L\left(\Lambda_{(s)}\right)} q^{L_{0}}$ in terms of the multivariable Appell sums $\mu_{n, \ell}$, the Mordell integral $h$, and the Dedekind $\eta$-function. In what follows and throughout, for $c \in \mathbb{C}$, we use the notation $\mathbf{c}:=(c, c, \ldots, c) \in \mathbb{C}^{N}$, $N \in \mathbb{N}$.

Proposition 2.1. For $m \in \mathbb{N}, m \geq 2$, and $s \in \mathbb{Z}$, we have

$$
\begin{aligned}
\operatorname{tr}_{L\left(\Lambda_{(s)}\right)} q^{L_{0}}=i^{-m+1} & \frac{\eta^{2 m}\left(-\frac{1}{2 \tau}\right)}{\eta^{2 m+1}\left(-\frac{1}{\tau}\right)} \sqrt{\frac{1}{(m-1)}} e^{-\frac{\pi i s}{m-1}+\frac{\pi i(m-2)}{4(m-1) \tau}+\pi i \tau\left(\frac{s^{2}}{m-1}+\frac{m-2}{12}\right)} \\
& \times \sum_{r=0}^{m-2} e^{-\frac{2 \pi i r s}{m-1}} \mu_{m-1, r}\left(-\frac{1}{2 \tau}, \frac{-\mathbf{1}}{\mathbf{2 \tau}} ;-\frac{1}{\tau}\right) \\
& +\frac{\sqrt{-i \tau}}{2} q^{\frac{m-2}{24}} \frac{\eta^{2 m}\left(-\frac{1}{2 \tau}\right)}{\eta^{2 m+1}\left(-\frac{1}{\tau}\right)} h(s \tau ;(m-1) \tau) .
\end{aligned}
$$

To prove Proposition 2.1, we will make use of various modular and elliptic transformation laws satisfied by the functions $\mu_{n}$. In particular, from the multivariable Appell function $\mu_{n}$, Zwegers 22 formed a nonholomorphic function $\widehat{\mu}_{n}$ and established modular transformation properties of this "completed" function $\widehat{\mu}_{n}$. To be more precise, define

$$
\widehat{\mu}_{n}(u, v)=\widehat{\mu}_{n}(u, v ; \tau):=\mu_{n}(u, v ; \tau)-\frac{i}{2} R\left(u-|v|-\frac{n+1}{2} ; n \tau\right) .
$$


Here the real-analytic function $R$ is defined by

$$
R(u)=R(u ; \tau):=\sum_{\nu \in \frac{1}{2}+\mathbb{Z}}\{\operatorname{sgn}(\nu)-E((\nu+a) \sqrt{2 y})\}(-1)^{\nu-\frac{1}{2}} e^{-\pi i \nu^{2} \tau-2 \pi i \nu u},
$$

where $y:=\operatorname{Im}(\tau), a:=\operatorname{Im}(u) / \operatorname{Im}(\tau)$, and the function $E$ is defined by

$$
E(u):=2 \int_{0}^{u} e^{-\pi u^{2}} d u
$$

The following proposition states some modular and elliptic transformation laws for the functions $R, \widehat{\mu}_{n}$, and $h$ as established by Zwegers [21, 22]. The function $\widehat{\mu}_{n, \ell}$ appearing in Proposition 2.2 below is defined as in (2.2), with $\mu_{n, \ell}$ replaced by $\widehat{\mu}_{n, \ell}$.

Proposition 2.2. Let $u, v \in \mathbb{C}, \tau \in \mathbb{H}, \lambda_{1}, \nu_{1} \in \mathbb{Z}$ and $\lambda_{2}, \nu_{2} \in \mathbb{Z}^{n}$ such that $\lambda_{1}-\left|\lambda_{2}\right| \in n \mathbb{Z}$. Then the following are true:

$$
\begin{aligned}
& \text { (1) } \widehat{\mu}_{n}(u, v)=(-1)^{\lambda_{1}+\left|\lambda_{2}\right|+\nu_{1}+\left|\nu_{2}\right|} e^{-\frac{2 \pi i}{n}\left(\lambda_{1}-\left|\lambda_{2}\right|\right)(u-|v|)} q^{-\frac{1}{2 n}\left(\lambda_{1}-\left|\lambda_{2}\right|\right)^{2}} \\
& \times \widehat{\mu}_{n}\left(u+\lambda_{1} \tau+\nu_{1}, v+\lambda_{2} \tau+\nu_{2}\right), \\
& \text { (2) } \widehat{\mu}_{n, \ell}(u, v ; \tau)=i^{n+1} \sqrt{\frac{i}{n \tau}} e^{\frac{\pi i}{n \tau}(u-|v|)^{2}} \sum_{r=0}^{n-1} e^{\frac{2 \pi i r \ell}{n}} \widehat{\mu}_{n, r}\left(-\frac{u}{\tau},-\frac{v}{\tau} ;-\frac{1}{\tau}\right) \text {, } \\
& \text { (3) } R(u+1)=-R(u) \text {, } \\
& \text { (4) } R(u ; \tau)=-\frac{1}{\sqrt{-i \tau}} e^{\frac{\pi i u^{2}}{\tau}}\left(R\left(\frac{-u}{\tau} ;-\frac{1}{\tau}\right)-h\left(\frac{-u}{\tau} ;-\frac{1}{\tau}\right)\right) \text {, } \\
& \text { (5) } h(u ; \tau)=\frac{1}{\sqrt{-i \tau}} e^{\frac{\pi i u^{2}}{\tau}} h\left(\frac{u}{\tau} ;-\frac{1}{\tau}\right) \text {. }
\end{aligned}
$$

We next give a proposition which relates the function $R$ at the arguments $\frac{\tau}{n}$ and $n \tau$. Its proof just requires the explicit definition of $R$ as given in (2.5).

Proposition 2.3. For $n \in \mathbb{N}, u \in \mathbb{C}, \tau \in \mathbb{H}$, we have

$R\left(u ; \frac{\tau}{n}\right)=\sum_{\ell=0}^{n-1} q^{-\frac{1}{2 n}\left(\ell-\frac{n-1}{2}\right)^{2}} e^{-2 \pi i\left(\ell-\frac{n-1}{2}\right)\left(u+\frac{1}{2}\right)} R\left(n u+\left(\ell-\frac{n-1}{2}\right) \tau+\frac{n-1}{2} ; n \tau\right)$.

We are now ready to prove Proposition 2.1.

Proof of Proposition 2.1. It is not hard to see that one can rewrite the KacWakimoto characters as

$$
\operatorname{tr}_{L\left(\Lambda_{(s)}\right)} q^{L_{0}}=i(-2)^{m} q^{\frac{m-2}{24}} \frac{\eta^{2 m}(2 \tau)}{\eta^{2 m+1}(\tau)} \mu_{m-1}\left(\frac{1}{2}-s \tau, \frac{\mathbf{1}}{\mathbf{2}} ; \tau\right) .
$$


Next, using (2.4) and the transformation laws in Proposition 2.2, we rewrite (for $n \in \mathbb{N})$

$$
\begin{aligned}
& \mu_{n}\left(\frac{1}{2}-s \tau, \frac{\mathbf{1}}{\mathbf{2}} ; \tau\right) \\
& =i^{n+1} \sqrt{\frac{i}{n \tau}} e^{\frac{-\pi i s}{n}+\frac{\pi i(n-1)^{2}}{4 n \tau}+\frac{\pi i s^{2} \tau}{n}} \sum_{r=0}^{n-1} e^{-\frac{2 \pi i r s}{n}} \mu_{n, r}\left(-\frac{1}{2 \tau},-\frac{\mathbf{1}}{\mathbf{2} \tau} ;-\frac{1}{\tau}\right) \\
& +\frac{i^{n}}{2} \sqrt{\frac{i}{n \tau}} e^{-\frac{\pi i s}{n}+\frac{\pi i(n-1)^{2}}{4 n \tau}+\frac{\pi i s^{2} \tau}{n}} \sum_{r=0}^{n-1} e^{-\frac{2 \pi i r s}{n}}(-1)^{r} e^{\frac{\pi i r^{2}}{n \tau}} e^{-\frac{2 \pi i r}{n}\left(\frac{n-1}{2 \tau}\right)} \\
& \quad \times R\left(\frac{n-1}{2 \tau}-\frac{r}{\tau}-\frac{n+1}{2} ;-\frac{n}{\tau}\right) \\
& -\frac{i}{2}(-1)^{n} \sqrt{\frac{i}{n \tau}} e^{\frac{\pi i s^{2} \tau}{n}} R\left(\frac{s}{n} ;-\frac{1}{n \tau}\right)+\frac{i}{2}(-1)^{n} \sqrt{\frac{i}{n \tau}} e^{\frac{\pi i s^{2} \tau}{n} h} h\left(\frac{s}{n} ;-\frac{1}{n \tau}\right) .
\end{aligned}
$$

To further simplify (2.7) we apply Proposition 2.3 with $u=s / n$ and replace $\tau$ by $-1 / \tau$ to find that $R\left(\frac{s}{n} ;-\frac{1}{n \tau}\right)$ equals

$$
\begin{aligned}
-i e^{-\frac{\pi i s}{n}+\frac{\pi i(n-1)^{2}}{4 n \tau}-\frac{\pi i n}{2}} \sum_{r=0}^{n-1}(-1)^{r} e^{-\frac{2 \pi i r s}{n}+\frac{\pi i r^{2}}{n \tau}-\frac{\pi i r(n-1)}{n \tau}} & \\
& \times R\left(\left(r-\frac{n-1}{2}\right)\left(-\frac{1}{\tau}\right)-\frac{n+1}{2} ;-\frac{n}{\tau}\right) .
\end{aligned}
$$

Using this, we obtain from (2.7) that $\mu_{n}\left(\frac{1}{2}-s \tau, \frac{\mathbf{1}}{2} ; \tau\right)$ equals

$$
\begin{gathered}
i^{n+1} \sqrt{\frac{i}{n \tau}} e^{\frac{-\pi i s}{n}+\frac{\pi i(n-1)^{2}}{4 n \tau}+\frac{\pi i s^{2} \tau}{n}} \sum_{r=0}^{n-1} e^{-\frac{2 \pi i r s}{n}} \mu_{n, r}\left(-\frac{1}{2 \tau},-\frac{\mathbf{1}}{\mathbf{2} \tau} ;-\frac{1}{\tau}\right) \\
+\frac{i}{2}(-1)^{n} \sqrt{\frac{i}{n \tau}} e^{\frac{\pi i s^{2} \tau}{n}} h\left(\frac{s}{n} ;-\frac{1}{n \tau}\right) .
\end{gathered}
$$

Inserting (2.8) into (2.6) (with $n=m-1$ ), using Proposition 2.2 (5), and the transformation law of the $\eta$-function, $\eta(-1 / \tau)=\sqrt{-i \tau} \eta(\tau)$ gives the claim.

\section{Asymptotic behavior of the KaC-Wakimoto Characters}

In this section we determine the asymptotic behavior of the Kac-Wakimoto characters $\operatorname{tr}_{L\left(\Lambda_{(s)}\right)} q^{L_{0}}$ by making use of Proposition 2.1. and analyzing the behavior of the functions $\eta, \mu_{n, r}$, and $h$ in Proposition 3.1 and Proposition 3.2 .

Proposition 3.1. As $t \rightarrow 0^{+}$, for $n \in \mathbb{N}, s \in \mathbb{Z}$, and $0 \leq r \leq n-1$, we have that

$$
\begin{aligned}
& \text { (1) } \frac{\eta^{2 n+2}\left(\frac{i}{2 t}\right)}{\eta^{2 n+3}\left(\frac{i}{t}\right)}=e^{\frac{\pi}{12 t}(n+2)}\left(1+O\left(e^{-\frac{\pi}{t}}\right)\right), \\
& \text { (2) } \mu_{n, r}\left(\frac{i}{2 t}, \frac{\boldsymbol{i}}{\mathbf{2 t}} ; \frac{i}{t}\right) \sim\left(\begin{array}{c}
n+1 \\
r+1
\end{array}\right) i^{n} e^{\frac{\pi}{n t}\left(r^{2}+(1-n) r\right)-\frac{\pi}{2 t}-\frac{\pi n}{4 t}} .
\end{aligned}
$$


Proof of Proposition 3.1. Proposition 3.1 (1) is obvious. To prove Proposition 3.1 (2), we rewrite

$$
\mu_{n, r}\left(\frac{\tau}{2}, \frac{\boldsymbol{\tau}}{\mathbf{2}} ; \tau\right)=\frac{(-1)^{r} q^{-\frac{r^{2}}{2 n}+\frac{r(n-1)}{2 n}+\frac{1}{4}+\frac{r}{2}}}{\vartheta^{n}\left(\frac{\tau}{2} ; \tau\right)} \sum_{k \in \mathbb{Z}^{n}} \frac{(-1)^{|k|} q^{\frac{1}{2}|| k||^{2}+|k|}}{1-q^{|k|+r+\frac{1}{2}}} .
$$

We first determine the smallest exponent in $q$ in the Lerch sum. For this, we rewrite it as

$$
\begin{aligned}
\sum_{k \in \mathbb{Z}^{n}} \frac{(-1)^{|k|} q^{\frac{1}{2}|| k||^{2}+|k|}}{1-q^{|k|+r+\frac{1}{2}}}= & \sum_{\substack{k \in \mathbb{Z}^{n} \\
|k|+r+\frac{1}{2}>0 \\
j \geq 0}}(-1)^{|k|} q^{\frac{1}{2}|| k||^{2}+|k|+j\left(|k|+r+\frac{1}{2}\right)} \\
& -\sum_{\substack{k \in \mathbb{Z}^{n} \\
|k|+r+\frac{1}{2}<0 \\
j \geq 1}}(-1)^{|k|} q^{\frac{1}{2}|| k||^{2}+|k|-j\left(|k|+r+\frac{1}{2}\right)} .
\end{aligned}
$$

We first treat the first sum on the right-hand side of (3.2). For the exponent to be minimal one requires that $j=0$ and this contribution is given by

$$
\sum_{\substack{k \in \mathbb{Z}^{n} \\|k|+r+\frac{1}{2}>0}}(-1)^{|k|} q^{\frac{1}{2}|| k||^{2}+|k|}=q^{-\frac{n}{2}}(-1)^{n} \sum_{\substack{k \in \mathbb{Z}^{n} \\|k|+r+\frac{1}{2}-n>0}}(-1)^{|k|} q^{\frac{1}{2}|| k||^{2}} .
$$

The minimal exponent occurs if $n-r$ of the $k_{i}$ are 1 and the remaining $k_{i}$ are 0 . This gives the contribution $(-1)^{r}\left(\begin{array}{c}n \\ r\end{array}\right) q^{-\frac{r}{2}}$. Similarly, the second sum on the right-hand side of (3.2) has minimal exponent for $j=1$ and this contribution is given by

$$
-q^{-r-\frac{1}{2}} \sum_{\substack{k \in \mathbb{Z}^{n} \\|k|+r+\frac{1}{2}<0}}(-1)^{|k|} q^{\frac{1}{2}|| k \|^{2}} .
$$

The exponent is minimal if $r+1$ of the $k_{i}$ are -1 and the remaining $k_{i}$ are 0 . This gives the contribution $(-1)^{r}\left(\begin{array}{c}n \\ r+1\end{array}\right) q^{-\frac{r}{2}}$. Combining the terms gives that the minimal exponent is $-\frac{r}{2}$ and this term has a weighting of $(-1)^{r}\left(\begin{array}{l}n+1 \\ r+1\end{array}\right)$.

Now one easily obtains the claim, using that

$$
\vartheta\left(\frac{i}{2 t} ; \frac{i}{t}\right) \sim-i e^{\frac{\pi}{4 t}}
$$

Finally, we analyze the behavior of the functions $h($ sit; nit), which one can see by Proposition 2.1. Proposition 3.1 (with $n=m-1$ ), and Proposition 3.2 below essentially determines the asymptotic expansion of the Kac-Wakimoto series beyond the main term.

Proposition 3.2. For $s \in \mathbb{Z}, n, N \in \mathbb{N}$, and $t \in \mathbb{R}^{+}$, we have as $t \rightarrow 0^{+}$,

$$
h(\text { sit; nit })=\sum_{j=0}^{N} a_{j}(n, s) t^{j}+O\left(t^{N+1}\right),
$$

where the coefficients $a_{j}$ are defined in (1.3). Moreover, for $n=2 s$, we have

$$
h(\text { sit } ; 2 s i t)=e^{-\frac{\pi s t}{2}}=e^{-\frac{\pi n t}{4}} .
$$


Remarks. 1) One can also rewrite the asymptotic expansion above for $h$ (sit; nit) in terms of the Riemann-zeta function and the Hurwitz-zeta function, using the identity (see [15], equation (3.15))

$$
E_{2 j}=\frac{2(2 j) !(-1)^{j}}{\pi^{2 j+1} 4^{j}}\left(4^{j}\left(1-2 \cdot 4^{j}\right) \zeta(2 j+1)+\zeta\left(2 j+1, \frac{1}{4}\right)\right) .
$$

2) The implied constant in Proposition 3.2 depends on $s, n$, and $N$.

Proof of Proposition 3.2 . We first consider the general case. By definition, we have

$$
H(t):=h(\text { sit } ; \text { nit })=\int_{\mathbb{R}} \frac{e^{-2 \pi i s t x-\pi n t x^{2}}}{\cosh (\pi x)} d x .
$$

It is not hard to see that $H$ is infinitely many times differentiable. By Taylor's Theorem we have that

$$
H(t)=T_{N}(t)+R_{N}(t),
$$

where $T_{N}$ is the $N$ th Taylor polynomial at 0 , and we use the remainder $R_{N}$ in the Lagrange form

$$
R_{N}(t):=\frac{h^{(N+1)}(c)}{(N+1) !} t^{N+1}
$$

with $c \in(0, t)$.

To prove Proposition 3.2, we will first explicitly determine $T_{N}$ and then show that $R_{N}(t)=O\left(t^{N+1}\right)$. We first observe that for $\ell \in \mathbb{N}_{0}$,

$$
H^{(\ell)}(t)=\int_{\mathbb{R}} \frac{e^{-2 \pi i s t x-\pi n t x^{2}}}{\cosh (\pi x)}\left(-2 \pi i s x-n \pi x^{2}\right)^{\ell} d x,
$$

where we justify interchanging the order of integration and differentiation by dominated convergence. We now write $T_{N}(t)=\sum_{j=0}^{N} a_{j}(n, s) t^{j}$ and show that the coefficients $a_{j}$ have the representation given in (1.3). From (3.4) we obtain, using the Binomial Theorem, that

$a_{j}(n, s)=\frac{(-\pi)^{j}}{j !} \int_{\mathbb{R}} \frac{\left(n x^{2}+2 i s x\right)^{j}}{\cosh (\pi x)} d x=\frac{(-\pi)^{j}}{j !} \sum_{\ell=0}^{j}\left(\begin{array}{l}j \\ \ell\end{array}\right) n^{\ell}(2 i s)^{j-\ell} \int_{\mathbb{R}} \frac{x^{j+\ell}}{\cosh (\pi x)} d x$.

Using the known identity for Euler numbers (see [9], for example),

$$
\int_{\mathbb{R}} \frac{x^{\ell}}{\cosh (\pi x)} d x=(-2 i)^{-\ell} E_{\ell}
$$

gives the shape of the coefficients $a_{j}(n, s)$ as claimed in the proposition.

We will next prove that $R_{N}(t)=O\left(t^{N+1}\right)$, which is equivalent to showing that $H^{(N+1)}(c)$ (recall that $c \in(0, t)$ ) can be uniformly bounded (i.e. independent of $t$ ). Proceeding as in (3.5), we obtain

$$
\left|H^{(N+1)}(c)\right| \leq \pi^{N+1} \sum_{\ell=0}^{N+1}\left(\begin{array}{c}
N+1 \\
\ell
\end{array}\right) n^{\ell}(2|s|)^{N+1-\ell} \int_{\mathbb{R}} \frac{|x|^{N+1+\ell}}{\cosh (\pi x)} d x<\infty
$$


which is independent of $t$. To justify the finiteness of (3.6), note that for $n \in \mathbb{N}_{0}$,

$$
\begin{aligned}
& \int_{\mathbb{R}} \frac{|x|^{2 n}}{\cosh (\pi x)} d x=(-1)^{n} 2^{-2 n} E_{2 n}, \\
& \int_{\mathbb{R}} \frac{|x|^{2 n+1}}{\cosh (\pi x)} d x=2\left(\int_{0}^{1}+\int_{1}^{\infty}\right) \frac{x^{2 n+1}}{\cosh (\pi x)} d x \leq 2+(-1)^{n+1} 2^{-2 n-1} E_{2 n+2} .
\end{aligned}
$$

We finally consider the special case $n=2 s$. Here the situation simplifies if one instead works with the inverted function $h$. To be more precise, Proposition 2.2 (5) gives that

$$
h(\text { sit } ; 2 \text { sit })=\frac{1}{\sqrt{2 s t}} e^{-\frac{\pi s t}{2}} h\left(\frac{1}{2} ;-\frac{1}{2 s i t}\right) .
$$

Inserting the definition of $h$ gives

$$
h\left(\frac{1}{2} ;-\frac{1}{2 s i t}\right)=\int_{\mathbb{R}} \frac{e^{-\frac{\pi x^{2}}{2 s t}-\pi x}}{\cosh (\pi x)} d x=\int_{\mathbb{R}} e^{-\frac{\pi x^{2}}{2 s t}} d x=\sqrt{2 s t},
$$

so that $h($ sit $; 2 s i t)=e^{-\frac{\pi s t}{2}}$.

Theorem 1.1 now follows easily from Proposition 2.1, Proposition 3.1 (with $n=$ $m-1$ ), and Proposition 3.2 .

\section{ACKNOWLEDGEMENTS}

The authors thank Don Zagier for fruitful conversations. Moreover they thank Karl Mahlburg for useful comments on an earlier version of the paper. The first author is partially supported by NSF grant DMS-0757907 and by the Alfried Krupp Prize for Young University Teachers of the Krupp Foundation. The second author is supported by NSF grant DMS-1049553.

\section{REFERENCES}

[1] R. Borcherds, Monstrous moonshine and monstrous Lie superalgebras, Invent. Math. 109 (1992), 405-444. MR1172696 (94f:11030)

[2] K. Bringmann, Asymptotics for rank partition functions, Trans. Amer. Math. Soc. 361 (2009), 3483-3500. MR2491889(2010g:11175)

[3] K. Bringmann and B. Kane, Inequalities for differences of Dyson's rank for all odd moduli, Math. Res. Lett. 17 (2010), 927-942. MR2727619(2011i:11155)

[4] K. Bringmann and J. Lovejoy, Overpartitions and class numbers of binary quadratic forms, Proc. Nat. Acad. Sci. USA 106 (2009), 5513-5516. MR2504957 (2010e:11031)

[5] K. Bringmann and K. Ono, The $f(q)$ mock theta function conjecture and partition ranks, Invent. Math. 165 (2006), 243-266. MR2231957 (2007e:11127)

[6] K. Bringmann and K. Ono, Dyson's ranks and Maass forms, Ann. of Math. (2) 171 (2010), 419-449. MR2630043(2011e:11165)

[7] K. Bringmann and K. Ono, Some characters of Kac and Wakimoto and nonholomorphic modular functions, Math. Annalen 345 (2009), 547-558. MR2534107(2010f:11061)

[8] J. Conway and S. Norton, Monstrous moonshine, Bull. London Math. Soc. 11 (1979), 308339. MR.554399 (81j:20028)

[9] A. Erdelyi, W. Magnus, F. Oberhettinger, and F. Tricomi, Higher transcendental functions, Vol. III, based on notes left by Harry Bateman, reprint of the 1955 original, Robert E. Krieger Publishing Co., Inc., Melbourne, Fla., 1981. MR698781 (84h:33001c)

[10] A. Folsom, Kac-Wakimoto characters and universal mock theta functions, Trans. Amer. Math. Soc. 363 (2011), 439-455. MR2719689 (2011h:11044)

[11] B. Gordon and R. McIntosh, A survey of mock theta functions I, preprint.

[12] V. Kac, Infinite-dimensional Lie algebras and the Dedekind $\eta$-function, Funct. Anal. Appl. 8 (1974), 68-70. MR0374210(51:10410) 
[13] V. Kac, Infinite-dimensional Lie algebras, Third edition. Cambridge University Press, Cambridge, 1990. MR:1104219 (92k:17038)

[14] V. Kac and M. Wakimoto, Integrable highest weight modules over affine superalgebras and Appell's function, Comm. Math. Phys. 215 (2001), 631-682. MR1810948 (2001j:17017)

[15] T. Kim, Euler numbers and polynomials associated with zeta functions, Abstr. Appl. Anal. Art. ID 581582 (2008). MR2407279 (2009b:11039)

[16] J. Lepowsky and S. Milne, Lie algebraic approaches to classical partition identities, Advances in Mathematics 29 (1978), 15-59. MR501091 (82f:17005)

[17] J. Lepowsky and S. Milne, Lie algebras and classical partition identities, Proc. Nat. Acad. Sci. U.S.A. 75 (1978), 578-579. MR0491467 (58:10713)

[18] J. Lepowsky and R. Wilson, The Rogers-Ramanujan identities: Lie theoretic interpretation and proof, Proc. Nat. Acad. Sci. U.S.A. 78 (1981), 699-701. MR605423 (82a:17004)

[19] I. Macdonald, Affine root systems and Dedekind's $\eta$-function, Invent. Math. 15 (1972), 91143. MR0357528 (50:9996)

[20] K. Ono, Unearthing the visions of a master: harmonic Maass forms and number theory, Proceedings of the 2008 Harvard-MIT Current Developments in Mathematics Conference, International Press, Somerville, MA, 2009, 347-454. MR.2555930 (2010m:11060)

[21] S. Zwegers, Mock theta functions, Ph.D. Thesis, Universiteit Utrecht, 2002.

[22] S. Zwegers, Multivariable Appell functions, preprint, 2010.

Mathematical Institute, University of Cologne, 50931 Cologne, Germany

E-mail address: kbringma@math.uni-koeln.de

Department of Mathematics, Yale University, P.O. Box 208283, New Haven, ConNECTICUT 06520-8283

E-mail address: amanda.folsom@yale.edu 